\title{
Finite Element Modelling of Cold Formed Stainless Steel Columns
}

\begin{abstract}
M. Macdonald, J. Rhodes
This paper describes the results obtained from a finite element investigation into the load capacity of column members of lipped channel cross-section, cold formed from Type 304 stainless steel, subjected to concentric and eccentric compression loading. The main aims of this investigation were to determine the effects which the non-linearity of the stress-strain behaviour of the material would have on the column behaviour under concentric or eccentric loading. Stress-strain curves derived from tests and design codes are incorporated into non-linear finite element analyses of eccentrically loaded columns and the results obtained are compared with those obtained on the basis of experiments on stainless steel channel columns with the same properties and dimensions. Comparisons of the finite element results and the test results are also made with existing design specifications and conclusions are drawn on the basis of the comparisons.
\end{abstract}

Keywords: finite elements, stainless steel, cold forming.

\section{Introduction}

The mechanical properties of stainless steels are significantly different from those of carbon steel. Stainless steels display a pronounced response to cold working resulting in anisotropic, non-linear stress-strain behaviour and low proportional limits. The material properties of various stainless steels have been thoroughly investigated since the 1960s by a number of investigators, e.g. refs. [1, 2, 3, 4]. It has been generally concluded that the stress-strain behaviour of stainless steels can be best described by the Ramberg-Osgood model [5], and Hill's [6] modified form of the Ramberg-Osgood equation is used in design specifications.

The main design specification for cold formed stainless steel members in the USA is the ASCE specification [7] and in Europe, Eurocode 3: Part 1.4 [8] has been recently developed and is still under examination. The two codes use different approaches when dealing with the mechanical properties of the material. The ASCE code employs the modified form of the Ramberg-Osgood model to describe the stress-strain behaviour of a material, whereas the Eurocode relies for most purposes on the specification of a linear stress-strain law, with the yield strength taken as the $0.2 \%$ proof stress. Refs. $[9,10]$ show a comparison of the Eurocode and ASCE code load capacity predictions for lipped channel columns is illustrated. The simpler Eurocode analysis has been found to give reasonable estimates of concentrically loaded column strength without taking account of the non-linearity of the stress-strain curve. As part of a previous investigation ref. [11], a series of tensile tests were carried out on coupons cut from stainless steel lipped channel sections, and also on full sections, and the stress-strain characteristics are examined in this paper and incorporated into a non-linear finite element analysis.

\section{Mechanical properties of stainless steel lipped channel members}

In the formation of a profiled section, the cold working occurs in localised areas, with the material at the bends being strain hardened. Therefore the properties of the material vary throughout the cross-section where at the formed bends, higher yield and tensile strengths exist, leading to a more complex stress-strain relationship for cold formed members, and in particular, for stainless steel members. The level of increase of both yield and tensile strength is dependent on the ratio of corner radius to material thickness $(r / t)$. The cold formed lipped channels under investigation are of stainless steel, of cross-sections with small web, flange and lip dimensions and are considered to be thick and hence four corner bends are formed with small $r / t$ ratios $(<1)$. These four corners will have an effect on the stress-strain response of the material obtained from a full section test, which could then be compared to that obtained for virgin material from a standard tensile test. Also, most commercially available finite element programs allow for a non-linear analysis and hence the inclusion of the actual stress-strain data obtained from tensile testing and from existing theories.

The ASCE design specification adopts the modified form of the Ramberg-Osgood formula given by equation (1). It is a three-parameter equation for expressing the relationship between the stress and strain for stresses up to a value slightly greater than the yield strength of the material.

$$
\varepsilon=\frac{\sigma}{E}+K\left(\frac{\sigma}{E}\right)^{n},
$$

where $\varepsilon=$ unit strain

$$
\begin{aligned}
& \varepsilon=\text { unit stress }\left(\mathrm{N} / \mathrm{mm}^{2}\right) \\
& E=\text { modulus of elasticity }\left(\mathrm{N} / \mathrm{mm}^{2}\right)
\end{aligned}
$$

$K$ and $n$ are constants for a given curve, which are evaluated through two secant yield strength values for slopes of $0.7 E$ and $0.85 E$. Equation (1) was modified by Hill [6], and, instead of using secant yield strengths, $K$ and $n$ can be evaluated in terms of two yield strength values: (i) $\sigma_{1}$ at an offset $\varepsilon_{1}$; (ii) $\sigma_{2}$ at an offset $\varepsilon_{2}$.

Using the most common offset of 0.002 for the yield stress $\left(\sigma_{2}\right)$ and assuming that the modulus of elasticity $E$ is equal to the initial value $\mathrm{E}_{0}$, equation (1) becomes:

$$
\varepsilon=\frac{\sigma}{E_{0}}+0.002\left(\frac{\sigma}{\sigma_{y}}\right)^{n} .
$$

The ASCE design code makes use of this modified equation 2, and the three points on the stress-strain curve are defined as: (i) the origin; (ii) the point of $0.2 \%$ proof stress; 
(iii) another offset strength (e.g. $0.01 \%$ ). If these points are substituted into equation (2), then ' $n$ ' can be evaluated. The term ' $n$ ' is referred to in the ASCE design code as the plasticity factor. The accuracy of the above method is largely based on how well the analytical equation fits the stress-strain relationship of the material. The code lists for particular grades of stainless steel, tables of yield stress, tangent modulus and plasticity factors.

The results obtained for the stress-strain relationship from both virgin material and full section tensile tests will be used for comparison with the results obtained from the above ASCE Ramberg-Osgood approach and by a trial and error 'best fit' method using the experimental stress-strain curves. These will then be incorporated into a non-linear finite element analysis of eccentrically loaded stainless steel columns.

\section{Load capacity of stainless steel lipped channel columns subjected to combined bending and axial compression loading}

Rhodes et. al. [9, 10], investigated both concentric and eccentric loading of cold formed stainless steel lipped channel section columns. The findings showed that the relevant design codes refs $[7,8]$ provided very accurate predictions of load capacity for the concentric loading case using both virgin and full section material properties when compared to experimental results. A finite element analysis also produced a very accurate correlation to both the experimental results and the design code predictions. However, for shorter length eccentrically loaded columns, the design codes were very conservative in their prediction of load capacity using both virgin and full section material properties. It was concluded that the design codes' interaction formulae were inadequate in predicting the load capacity of short-to-medium length columns.

The Eurocode (8) interaction formula to determine the axial strength $N_{\mathrm{sd}}$ is given by equation (3).

$$
\frac{N_{\mathrm{sd}}}{\chi f_{y}\left(\frac{A}{\gamma_{M 1}}\right)}+\frac{\kappa N_{\mathrm{sd}} e}{\left(\frac{M_{n}}{\gamma_{M 1}}\right)} \leq 1,
$$

where all terms are defined in Ref. [9].

The ASCE [7] interaction formula to determine the axial strength $P_{u}$ is given by equation (4).

$$
\frac{P_{u}}{P_{n}}+\frac{P_{u} e}{M_{n}\left(1-\left(\frac{P_{u}}{P_{E}}\right)\right)} \leq 1.0 .
$$

where all terms are defined in Ref. [9].

Both equations produced very conservative estimates of load capacity and an attempt to improve the interaction formulae was proposed by Macdonald Ref. [12]. This modification to the interaction formulae involved replacing the linear moment capacity $M_{n}$ with the true moment capacity of the lipped channel cross-section $M_{\text {exp }}$ obtained from bending tests. Hence the ASCE interaction formula was modified as given by equation (5).

$$
\frac{P_{u}}{P_{n}}+\frac{P_{u} e}{M_{\exp }\left(1-\left(\frac{P_{u}}{P_{E}}\right)\right)} \leq 1.0 .
$$

The Eurocode interaction formula was modified as given by equation (6).

$$
\frac{N_{\mathrm{sd}}}{\chi f_{y}\left(\frac{A}{\gamma_{M 1}}\right)}+\frac{\kappa N_{\mathrm{sd}} e}{\left(\frac{M_{\mathrm{exp}}}{\gamma_{M 1}}\right)} \leq 1 .
$$

In both equations (5) and (6), $M_{\text {exp }}$ is the cross-section true moment capacity and the $0.2 \%$ proof stress is taken from the full section tensile test results, and all other terms are defined in Ref. [12].

\section{Finite element analysis}

A finite element analysis was then performed using the ANSYS software package to determine the load capacity of concentrically and eccentrically loaded columns. Two types of buckling analyses are available within the ANSYS package eigenvalue analysis and non-linear analysis. Both types of analyses were used, however, the eigenvalue analysis was used only to verify that the finite element model boundary conditions (i.e. column pin ends and eccentric loading) were accurate, as this type of analysis takes no account of material non-linearity. A full non-linear analysis was conducted using shell elements (ANSYS SHELL181) which are four-noded elements with six degrees of freedom at each node, i.e. translations in $x, y$ and $z$ directions, and rotations about $x, y$ and $z$ axes. Fig. 2 shows a typical displacement and boundary conditions plot for an eccentrically loaded column, while Fig. 3 shows a typical nodal stress plot.

For the eccentrically loaded columns, the non-linear material properties of the stainless steel were defined in ANSYS using the initial elastic modulus, Poisson's ratio and stress-strain data obtained from: (i) coupon tensile tests on material cut from section webs; (ii) full-section tensile tests; (iii) ASCE (Ramberg-Osgood) approach; (iv) 'best fit' stress-strain curves. For concentrically loaded columns, the virgin material properties were used.

The non-linear solution breaks the load up into a series of load increments that can be applied over several load steps. At the completion of each incremental solution, the program adjusts the stiffness matrix to reflect the non-linear changes in the structural stiffness before proceeding to the next load increment. For a non-linear buckling analysis to be accurate using ANSYS, it is necessary to set an initial imperfection in the structure being modelled. This was achieved by modelling a very small mid-span deflection which produced a very large radius of curvature for the lipped channel columns which would approximate any actual imperfections.

A parametric model was constructed by defining positions of keypoints to allow for easy alterations to the model, particularly for the variation in column length. A column half-model was modelled using appropriate symmetry commands that helped to reduce the considerable computer processing time. 


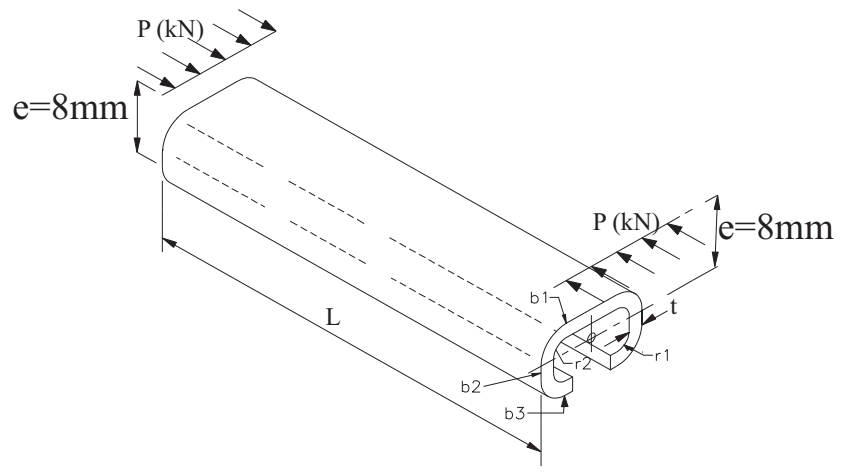

Fig. 1: Schematic diagram of eccentrically-loaded column test

\section{Experimental investigations}

\subsection{Tensile tests}

Fig. 1 shows a schematic diagram of a typical cold formed stainless steel lipped channel member under investigation. The member is commercially available with specimens cut from supplied lengths and all the specimens were accurately measured at a number of points, with the values averaged to obtain the finished dimensions, and all calculations were based on mid-line dimensions. The details are given in Table 1. In order to determine the material properties of the sections, tensile tests were set-up where the applied load and gauge specimen elongation were recorded continuously until fracture of the specimen occurred. The measured load and elongation were normalised to give a stress-strain relationship. Due to the anisotropy of stainless steel, a full analysis of the material properties would require tensile tests in the longitudinal and transverse directions, as well as compression tests in the same directions. Indeed, provision is made in the ASCE design code to enable use to be made of them in specific applications. However, compression tests were not carried out as there would be difficulty in establishing the true material properties of the material due to likely buckling effects. Also, transverse direction tensile tests could not be carried out because of the limitations in the geometry of the sections. Hence tensile testing was limited to the longitudinal direction.

Table 1: Average dimensions of lipped channel cross-section

\begin{tabular}{|c|c|c|c|c|c|}
\hline $\begin{array}{c}\text { Web } \\
b_{1}(\mathrm{~mm})\end{array}$ & $\begin{array}{c}\text { Flange } \\
b_{2}(\mathrm{~mm})\end{array}$ & $\begin{array}{c}\text { Lip } \\
b_{3}(\mathrm{~mm})\end{array}$ & $\begin{array}{c}\text { Thickness } \\
t(\mathrm{~mm})\end{array}$ & $\begin{array}{c}\text { Radius } \\
r_{1}(\mathrm{~mm})\end{array}$ & $\begin{array}{c}\text { Radius } \\
r_{2}(\mathrm{~mm})\end{array}$ \\
\hline 28.00 & 14.88 & 7.45 & 2.43 & 1.10 & 1.10 \\
\hline
\end{tabular}

All tensile tests were carried out in accordance with BSEN10002-1 [13]. Standard tensile tests were performed to ascertain the material properties of the stainless steel. Coupons were cut from the webs of the columns and tested to obtain the $0.2 \%$ proof stress and the modulus of elasticity.

Tensile tests were also performed on full sections to include the effects of the cold formed corners and from these tests, the $0.2 \%$ proof stress and the initial modulus of elasticity were determined.

For the standard coupons, a total of three specimens were tested and the average results were noted. For the full section tests, two specimens were tested and again, the average results were noted and shown in Table 2.

Table 2: Tensile test results: virgin material and full section (FS) mechanical properties

\begin{tabular}{|c|c|c|c|c|}
\hline $\begin{array}{c}\text { Thickness } \\
\mathrm{t}(\mathrm{mm})\end{array}$ & $\begin{array}{c}\text { Av. Virgin } \\
0.2 \% \text { P. S. } \\
\left(\mathrm{N} / \mathrm{mm}^{2}\right)\end{array}$ & $\begin{array}{c}\text { Av. Virgin } \\
\text { UTS } \\
\left(\mathrm{N} / \mathrm{mm}^{2}\right)\end{array}$ & $\begin{array}{c}\text { Av. FS } \\
0.2 \% \text { P. S. } \\
\left(\mathrm{N} / \mathrm{mm}^{2}\right)\end{array}$ & $\begin{array}{c}\text { Av. FS } \\
\text { UTS } \\
\left(\mathrm{N} / \mathrm{mm}^{2}\right)\end{array}$ \\
\hline 2.43 & 480 & 553 & 520 & 689 \\
\hline
\end{tabular}

The results obtained for the plasticity factors $\mathrm{n}$ from the ASCE design code, i.e. the modified form of the Ramberg-Osgood equation given by equation (2), are also detailed in ref. [11] and shown in Table 3 . Also shown in Table 3 are the plasticity factors obtained from a comparative plots/trial and error ('best fit') process using the stress-strain curves obtained from the tensile tests, as reported in Ref. [11].

Table 3: Plasticity factors

\begin{tabular}{|c|c|c|}
\hline Tensile test & $\begin{array}{c}n \\
\text { (ASCE) }\end{array}$ & $\begin{array}{c}\mathrm{n} \\
\text { (Best fit) }\end{array}$ \\
\hline Coupon & 3.80 & 6.22 \\
\hline Full section & 5.02 & 6.65 \\
\hline
\end{tabular}

\subsection{Compression tests}

In the experimental investigation a series of compression tests to failure were made on stainless steel columns of the lipped channel cross-section as described above. The specimen parameters investigated were as follows: column lengths varied from $222 \mathrm{~mm}$ to $1222 \mathrm{~mm}$ in increments of $100 \mathrm{~mm}$ (slenderness ratios varying from 42 to 234); twenty-two tests to failure (2 sets of columns, average results noted) were carried out with the loading applied concentric to the centroid of the cross-section to give pure axial compression; twenty-two tests to failure (2 sets of columns, average results noted) were carried out with the loading applied $8 \mathrm{~mm}$ eccentric to the centroid of the cross-section to give combined bending and axial compression loading.

Each length of column tested was cut to the specified length and then milled flat at each end to avoid any possible gripping problems. The end grips were designed such that they would hold the ends of the column and allow the loading to be applied at the required eccentricity through knife edges. The specimens were tested using a Tinius Olsen electro-mechanical testing machine, with the column vertical displacement and mid-span horizontal deflection measured during the tests using displacement transducers. Fig. 1 shows a schematic diagram of the column test configuration.

\subsection{Observations}

Fig. 4 shows the results obtained for the load capacity of concentrically loaded stainless steel lipped channel section columns from tests, design codes (using virgin material properties and full section properties) and from finite element analysis (using virgin material properties). The design code and finite element predictions show excellent correlation with the test results for all but the shortest of the columns. 


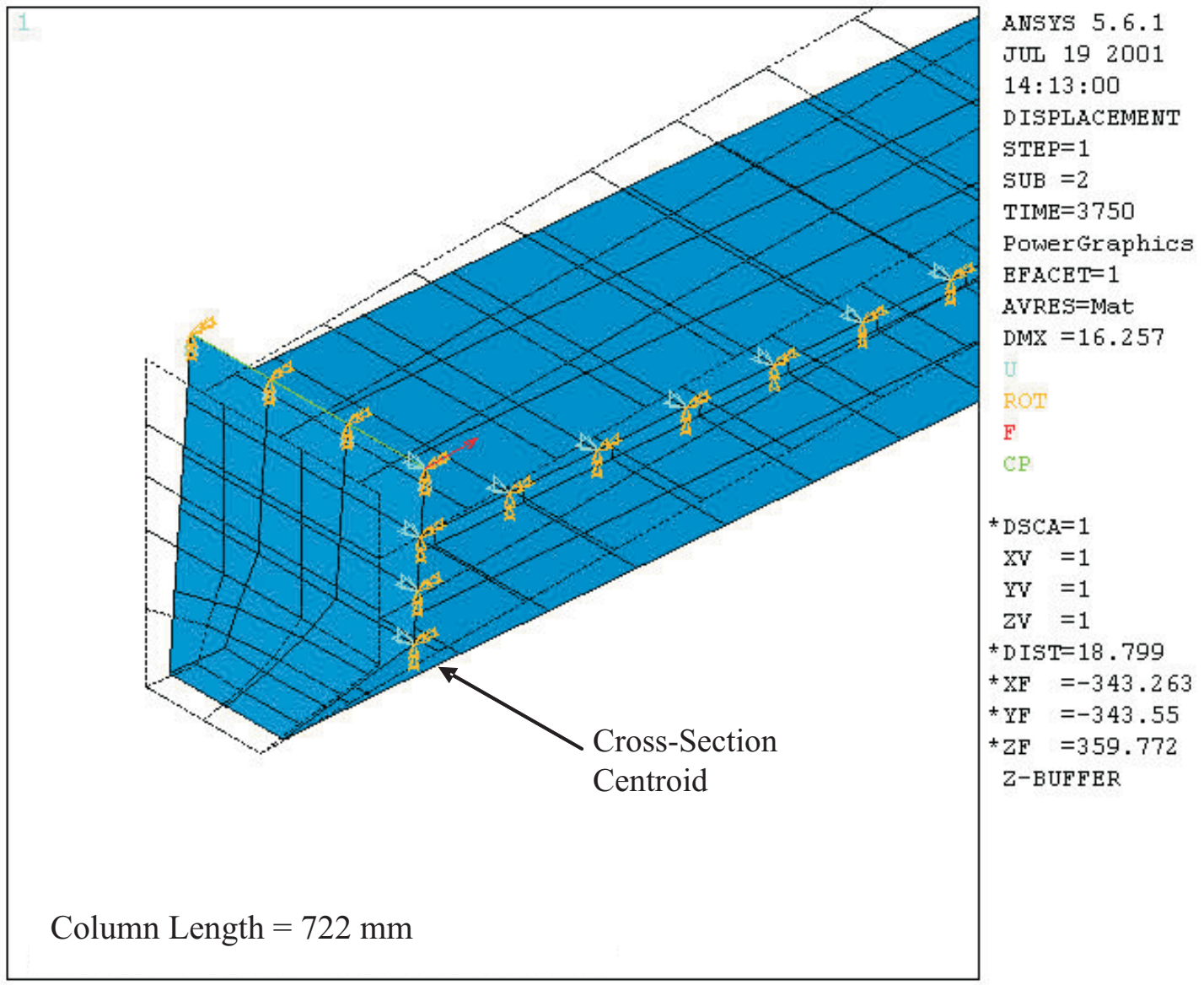

Fig. 2: Finite element plot of displacement and boundary conditions for eccentric column loading

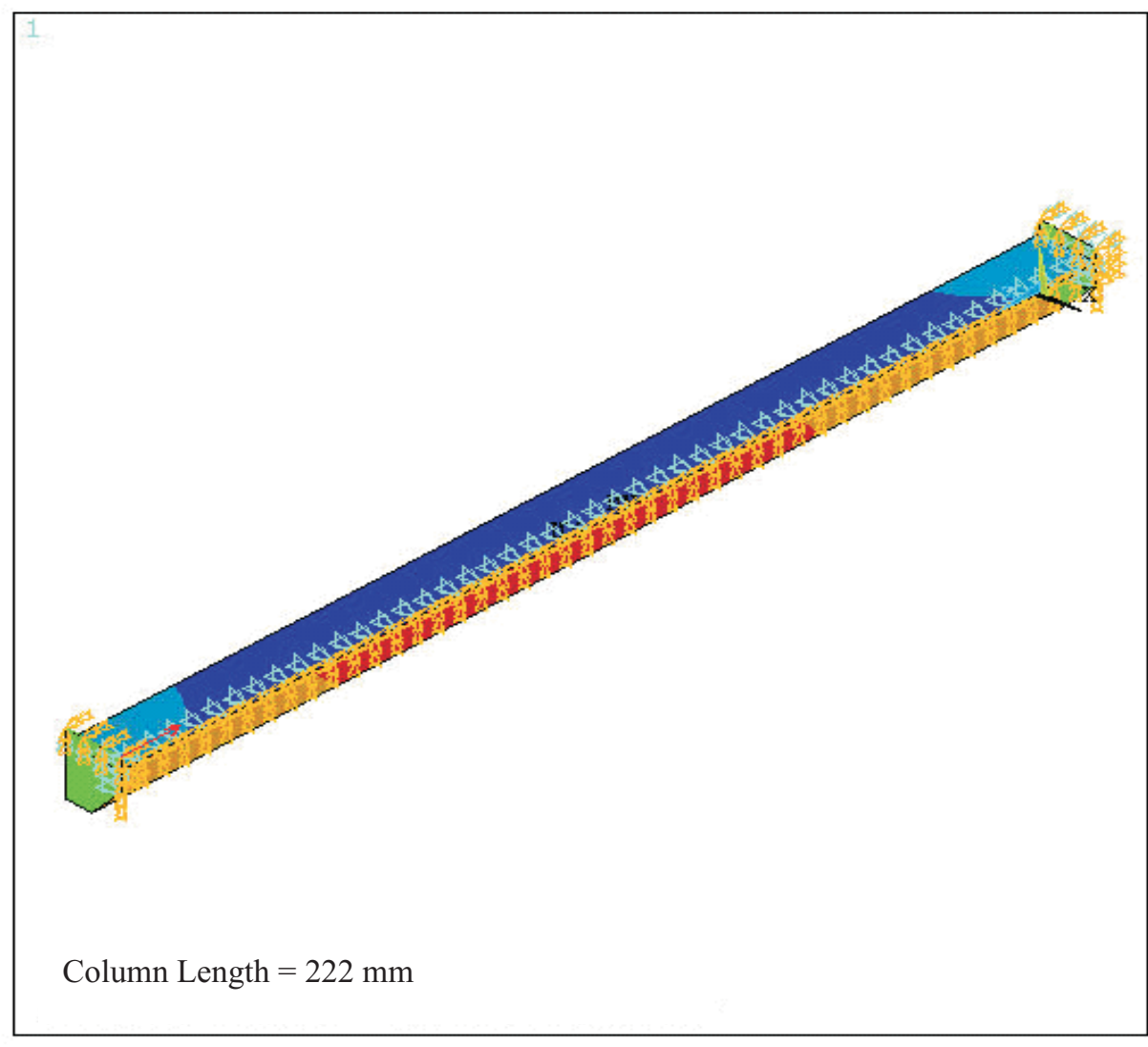

ANSYS 5.6 .1

JUL 192001

$13: 46: 06$

NODAL SOLUTION

$S T E P=1$

SUB $=9$

TIME $=13500$

$\mathrm{SZ}$

(AVG)

RSYS $=0$

PowerGraphics

EFACET $=1$

AVRES $=$ Mat

SMN $=-604.198$

$\operatorname{smx}=502.415$

RO'

$-604.198$

$-481.241$

$-358.284$

$-235.327$

$-112.37$

10.587

133.544

256.501

379.458

502.415

Fig. 3: Finite element plot of nodal stress and boundary conditions for eccentric column loading

(C) Czech Technical University Publishing House http://ctn.cvut.cz/ap/ 


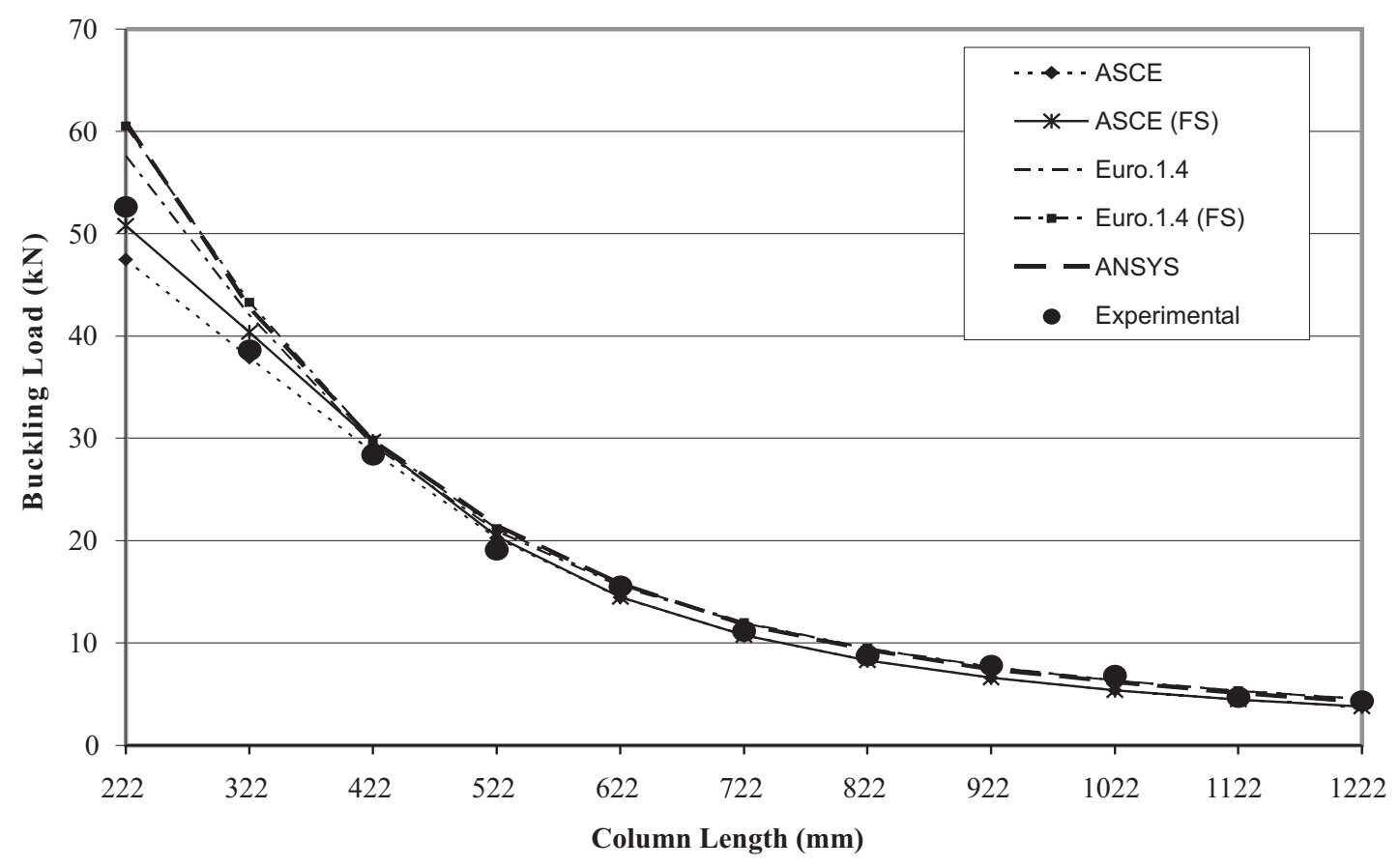

Fig. 4: Graph of load capacity v. column length: concentric loading (test/design codes/finite element analysis)

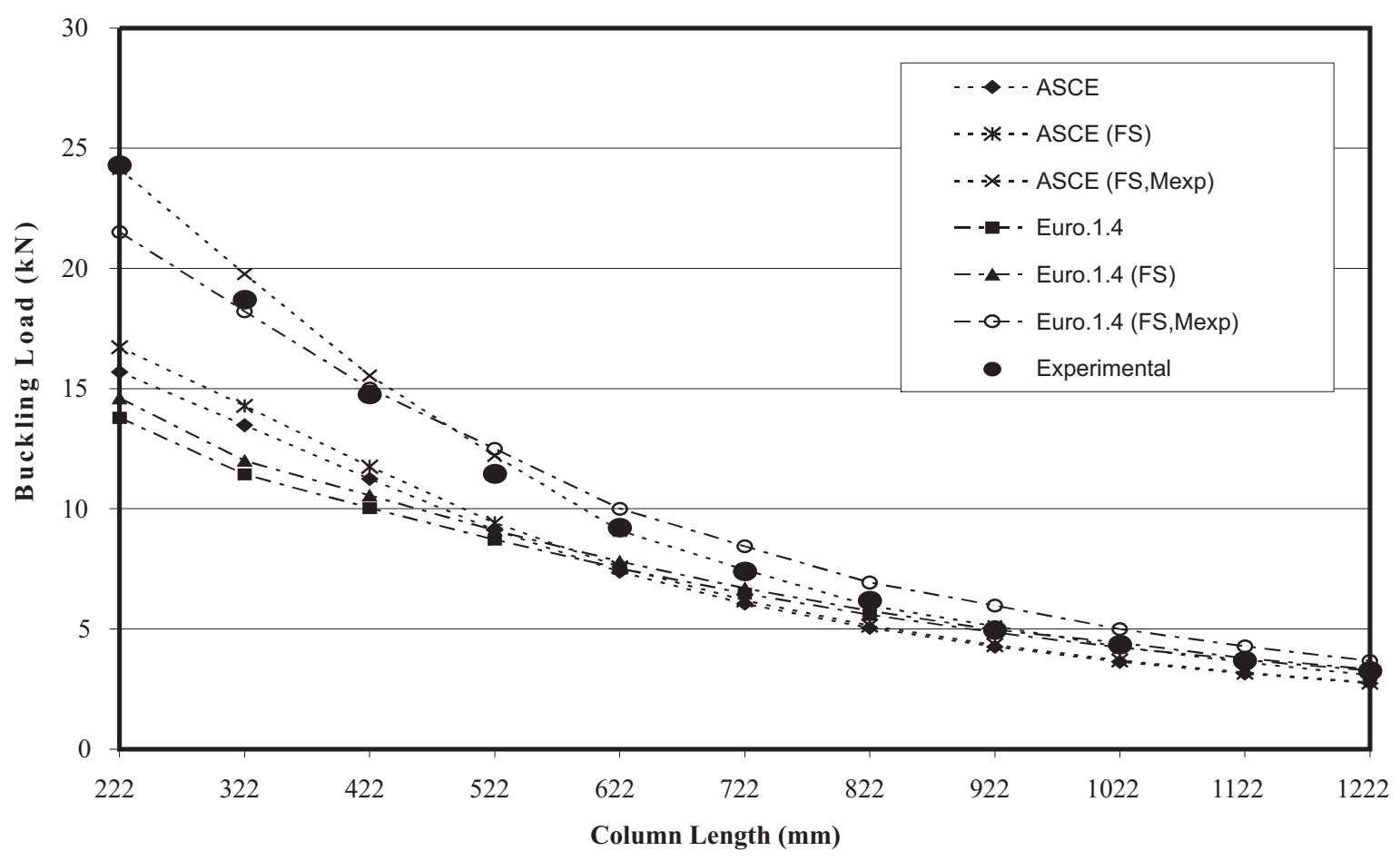

Fig. 5: Graph of load capacity v. column length: eccentric loading (test/design codes)

Fig. 5 shows the results obtained for the load capacity of eccentrically loaded stainless steel lipped channel section columns from tests, design codes (using virgin material properties and full section properties) and from modifications to the design codes as described by equations (5) and (6). All design code predictions show conservatism in prediction of load capacity for the shorter range of columns with improvements gained when full section properties are used and further improvements are gained in using the modified forms. The best correlation obtained was for columns where the modified design codes predicted accurate load capacities for all column lengths.

Fig. 6 shows the results obtained for the load capacity of eccentrically loaded stainless steel lipped channel section columns from tests and from finite element analysis using the various stress-strain curves described earlier. The predictions of the finite element analysis show an excellent correlation to the test results and a real improvement on the predictions of 


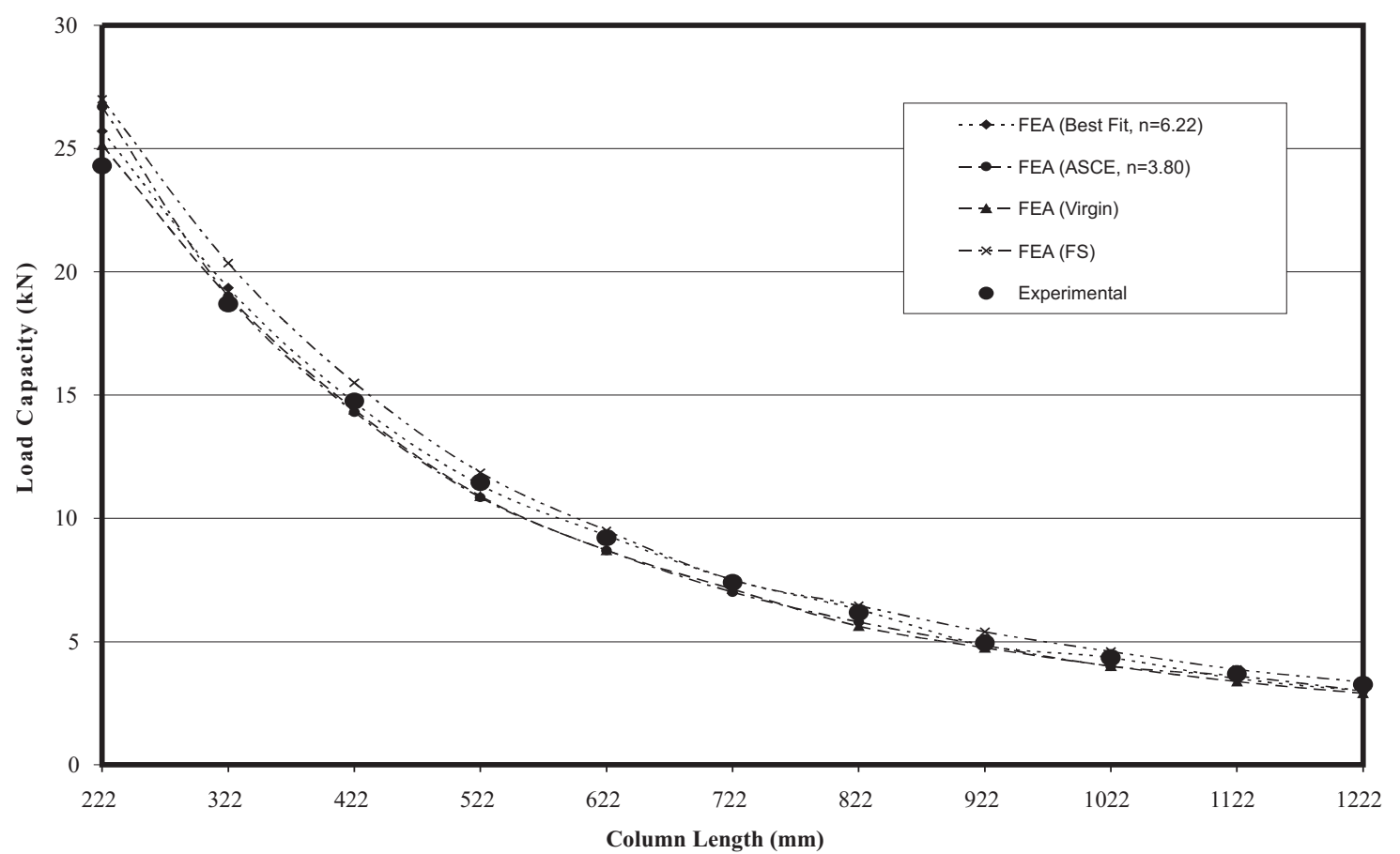

Fig. 6: Graph of load capacity v. column length: eccentric loading (test/finite element analysis)

the design codes. However, any differences between finite element predictions and test results are very slight and occur mainly for very short length columns.

\section{Conclusions}

The load capacity of short-to-medium length cold formed stainless steel lipped channels under pure axial compressive (concentric) loading was shown to be accurately predicted by the Eurocode 1.4 and the ASCE design code. A non-linear finite element analysis using virgin material stress-strain properties also provided accurate load predictions.

However, for eccentrically loaded columns with shorter lengths, the design codes predicted very conservative load capacities. Improvements were gained by employing enhanced stress-strain properties and by incorporating the full section moment capacity within the code interaction formulae.

Finally, it has been shown in this paper that finite element analysis can be used with a high level of confidence in predicting the load capacity of eccentrically loaded cold formed stainless steel, short-to-medium length columns of lipped channel section. This has been shown to be true for various stress-strain curves including that obtained from virgin material tensile tests.

\section{References}

[1] Johnson, A. L.: The Structural Performance of Austenitic Stainless Steel Members. Department of Structural Engineering, Report No 327. Cornell University, New York 1966.

[2] Wang, S. T.: Cold-Rolled Austenitic Steel: Material Properties and Structural Performance. Department of Structural En- gineering, Report No. 334. Cornell University, New York 1969.

[3] Wang, S. T., Errera, S. J. and Winter, G.: "Behaviour of Cold-Rolled Stainless Steel Members." Journal of the Structural Division, Proc. ASCE 101 (ST11), 1975, p. 2337-2357.

[4] Van Den Berg, G. J., Van Der Merwe, P.: "Prediction of Corner Mechanical Properties for Stainless Steels Due to Cold Forming." Paper Presented at the $11^{\text {th }}$ International Specialty Conference on Cold Formed Steel Structures, St.Louis, Missouri, (USA): October 1992.

[5] Ramberg, W., Osgood, W. R.: "Description of Stress-Strain Curves by Three Parameters." National Advisory Committee for Aeronautics (NACA), Technical Note No. 902, February 1943.

[6] Hill, H. N.: "Determination of Stress-Strain Relations from 'Offset' Yield Strength Values." National Advisory Committee for Aeronautics (NACA), Technical Note No. 927, February 1944.

[7] ANSI/ASCE-8-90: Specification for the Design of Cold-Formed Stainless Steel Structural Members. 1991.

[8] ENV 1993-1-3, Eurocode 3: Design of Steel Structures: Part 1.4: General Rules - Supplementary Rules for Stainless Steel. July 1996.

[9] Rhodes, J., Macdonald, M., McNiff, W.: "Buckling of Cold Formed Stainless Steel Columns under Concentric and Eccentric Loading." Proc. $15^{\text {th }}$ Int. Specialty Conference on Cold Formed Steel Structures, St Louis, Missouri (USA), October 2000.

[10] Rhodes, J., Macdonald, M., Kotelko,M., McNiff, W.: "Buckling of Cold Formed Stainless Steel Columns under Concentric and Eccentric Loading." Proc. $3^{\text {rd }}$ Int. 
Conference on Thin Walled Structures, Krakow (Poland), June 2001.

[11] Macdonald, M., Rhodes, J., Taylor, G. T.: "Mechanical Properties of Stainless Steel Lipped Channels." Proc. $15^{\text {th }}$ Int. Specialty Conference on Cold Formed Steel Structures, St Louis, Missouri (USA), October 2000.

[12] Macdonald, M.: "The Effects of Cold Forming on Material Properties and Post-Yield Behaviour of Structural Sections.” PhD Thesis. Glasgow Caledonian University, Glasgow (Scotland), January 2002.

[13] British Standards Institution: Tensile Testing of Metallic Materials. BSEN10002-1.
Martin Macdonald

Glasgow Caledonian University

School of Engineering, Science and Design

Cowcaddens Road

Glasgow, G4 0BA

Scotland, UK

Jim Rhodes

e-mail:jrhodes@mecheng.strath.ac.uk

University of Strathclyde

Department of Mechanical Engineering

75 Montrose Street

Glasgow, G1 1XJ, Scotland, UK 